\title{
Discharge characteristics and abatement of volatile organic compounds using plasma reactor packed with ceramic Raschig rings
}

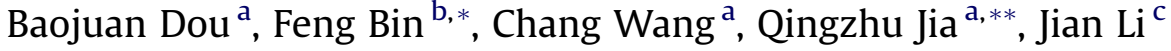 \\ ${ }^{a}$ College of Marine Science E Engineering, Tianjin University of Science E Technology, Tianjin 300457, PR China \\ ${ }^{\mathrm{b}}$ State Key Laboratory of High-temperature Gas Dynamics, Institute of Mechanics, Chinese Academy of Science, Beijing 100190, PR China \\ ${ }^{\mathrm{C}}$ College of Environmental \&' Energy Engineering, Beijing University of Technology, Beijing 100124, PR China
}

\section{A R T I C L E I N F O}

Article history:

Received 14 December 2012

Received in revised form 4 July 2013

Accepted 7 August 2013

Available online 23 August 2013

\section{Keywords:}

Non-thermal plasmas

Lissajous diagram

Discharge parameters

Ceramic Raschig rings

Toluene removal

\begin{abstract}
A B S T R A C T
Discharge characteristics and abatement of volatile organic compounds using plasma reactor packed with ceramic Raschig rings were investigated. It was found that the gap equivalent capacitance decreased with increasing voltage while the dielectric barrier equivalent capacitance increased initially and stabilized at about $700 \mathrm{pF}$. Compared with empty reactor, toluene removal was significantly enhanced by ceramic Raschig rings, $97 \%$ against $48 \%$. With respect to the energy yield in the presence of padding, the efficiency was remarkably improved up to $10 \mathrm{~g} / \mathrm{kWh}$, which was 2 times higher than that of $5 \mathrm{~g} / \mathrm{kWh}$ in the absence of padding with removal ratio exceeding $50 \%$.
\end{abstract}

Crown Copyright $\odot 2013$ Published by Elsevier B.V. All rights reserved.

\section{Introduction}

Volatile organic compounds (VOCs) have been extensively involved in many industrial processes, whereas such compounds are hazardous to human health, contributing to serious environmental problems such as the destruction of the ozone layer, photochemical smog and global warming [1,2]. The emission of VOCs has triggered increasing awareness focused on the development of abatement technologies to comply with the latest environmental regulations [3]. Traditional technologies for VOCs removal mainly include adsorption [4], thermal incineration [5], condensation [6] and infiltration [7] etc. All these technologies may have technical and/or economic disadvantages, hence, there is a motivation to develop method to control VOCs exhaust, which can be accepted both technically and economically [8]. As a late-model approach, nonthermal plasmas (NTP) processing has received considerable attentions owing to some unique advantages of rapidly reaction under ambient temperature, achievement of high electron energies within short residence time and easy operations. Previous studies have testified that NTP exhibits high removal efficiency by the

\footnotetext{
* Corresponding author. Tel./fax: +86 2227406842.

** Corresponding author.

E-mail addresses: binfeng@tju.edu.cn (F. Bin), jiaqingzhu@tust.edu.cn (Q. Jia).
}

mechanism of VOC decomposition [9-11], and it was considered as one of the most promising techniques to remove VOCs.

To further improve the electric field and power efficiency, dielectric materials have been considered as a key factor for the proper functioning of the NTP processing. A dielectric barrier with a high permittivity is highly desirable in order to generate plasma possessing high reactivity. $\mathrm{R}$. Li et al. [12] reported that the ratio of $\mathrm{CO}_{2}$ decomposition using $\mathrm{Ca}_{0.7} \mathrm{Sr}_{0.3} \mathrm{TiO}_{3}$ was much greater than commercial alumina and silica glass barriers, so as to obtain a conclusion that the $\mathrm{CO}_{2}$ decomposition was proportional to the permittivity of the dielectric barrier materials. Analogously, filling suitable dielectric materials shaped in appropriate geometries through discharge volume may be an effective approach to enhance the destruction efficiency for VOCs. For instance, M.A. Malik et al. [13] indicated that $\mathrm{C}_{2} \mathrm{HCl}_{3}$ destruction was significantly improved by packing the discharge gap with alumina pellets, compared with that in the absence of padding. According to Koichi Takaki et al. [14], the shapes of ceramic dielectric material pellet significantly influenced the performance of perfluoroethane $\left(\mathrm{C}_{2} \mathrm{~F}_{6}\right)$ removal, and the results showed that the energy yield of hollow cylindrical-shaped pellets was almost 1.5 times higher than that of spherical pellets.

In the NTP reactor, micro-discharges distributing throughout the discharge volume initiate the chemical reactions with the VOCs molecules decomposed by producing reactive species like ions, radicals and activated molecules [15]. During the discharge process, 
fundamental electricity characteristics such as voltage and current waveforms, discharge power, equivalent capacitance and so on, can be indirect macroscopical embodiments of various microcosmic chemical reactions. Nevertheless, it is difficult to measure these discharge parameters due to phase detuning of the current and voltage in the process of discharge. And there is very limited information available in the literature relating to the investigation of such parameters during the discharge process for VOCs removal.

In this study, coaxial cylindrical plasma reactor configuration was employed and corundum ceramic was chosen as the reactor material for its high permittivity to generate plasma possessing high reactivity. The paddings shaped in hollow cylindrical geometry were polarized when the reactor was powered, and an intense electric field was formed around edges and points, resulting in numerous micro-discharges. As a typical species of VOCs, toluene, which is widely used in industrial production processes, was adopted as the target contamination. The objective of this study is mainly to explore the decomposition of toluene by comparing main discharge parameters measured by $Q-V$ Lissajous diagram, in the presence and absence of ceramic Raschig rings packing in the coaxial cylindrical reactor of corundum ceramic. Meanwhile, the energy yield for toluene removal and the concentration of ozone detected in effluent gas were investigated.

\section{Experimental}

\subsection{Experimental setup}

Fig. 1 shows the schematic diagram of the experimental setup, consisting of continuous flow gas supplying system, electric and gaseous analytical systems, AC power supply ( $50 \mathrm{~Hz}, 0-100 \mathrm{kV}$, sine wave), and the NTP reactor. In the experiment, toluene was evaporated by bubbling with compressed air and then was allowed to pass through a mixing chamber for a thorough mixture with air before being introduced into the NTP reactor. The total gas flow rate and toluene concentration were adjusted by mass flow controllers (MFC-1 and MFC-4), which were fixed at $0.2 \mathrm{~m}^{3} / \mathrm{h}$ and $1200 \mathrm{mg} / \mathrm{m}^{3}$ respectively. Both the MFC- 1 and MFC- 4 employed to control the total gas flow rate are beneficial to determining the gas tightness. And, the gas flow rates of the two branches with and without toluene were controlled by MFC-3 and MFC-2 respectively. The coaxial cylindrical plasma reactor was made of corundum ceramic (99\% purity) tube with wall thickness of $3 \mathrm{~mm}$ and inner diameter of $19 \mathrm{~mm}$, wrapped by the copper mesh of $30 \mathrm{~cm}$ length as a ground electrode. A tungsten wire (1.65 $\mathrm{mm}$ in diameters) was employed as the inner discharge electrode, placed on the axis of the reactor. For comparison, ceramic Raschig rings (hollow cylindrical-shaped, $5 \mathrm{~mm}$ in outer diameter) were filled into the reactor during the experiment. Micro-discharges came into being when the sufficient high voltage was applied to the reactor, following a series of chemistry reactions.

\subsection{Gaseous analysis}

In the experiment, the concentrations of toluene from inlet and outlet were measured by using a gas chromatography (GC, Agilent 6890), equipped with a capillary column of HP-5 (diameter $0.32 \mathrm{~mm}$, film thickness $0.25 \mu \mathrm{m}$, length $30 \mathrm{~m}$ ) and a flame ionization detector (FID). The removal efficiency was calculated by the concentration change between the inlet and outlet concentrations of toluene. The concentration of ozone generated in the discharge from gaseous oxygen was accurately measured by the iodometric wet-chemistry method.

\subsection{Electrical measurements}

Oscilloscope (Tektronix 2014) was employed to measure the waves of the applied voltage and the current, as seen in Fig. 2. To investigate the discharge characteristics, the voltage applied to the NTP reactor was sampled by a $12500: 1(R 1 / R 2=250 \mathrm{M} \Omega / 20 \mathrm{k} \Omega)$ voltage divider, and the current was determined from the voltage drop across a shunt resistor $(R 3=10 \mathrm{k} \Omega)$ connected in series with the grounded electrode (Fig. 1). The experimental determination of the power dissipated in discharges has often proved to be difficult because in reality the power is consumed in a large number of short-lived microdischarges. We have used chargevoltage Lissajous method to determine the discharge power in the plasma reactors. The trick for the Lissajous figures is to use the time-integrated current, the charge, rather than trying to resolve individual microdischarge current peaks. Because the NTP reactor can be considered as a capacitor, the charge stored in the capacitor $C_{\mathrm{m}}$ is equal to that in the reactor. The charge stored in the capacitor is the product of capacitance and voltage, which can be directly read by the voltage between both ends of the capacitor. Here, a capacitor $\left(C_{\mathrm{m}}=0.33 \mu \mathrm{F}\right)$ was inserted between the NTP reactor and the ground to obtain the quantity of electricity (Fig. 1). When the switch is turned to the left, the value of electrical power provided to the reactor was measured by using the $Q-V$ Lissajous

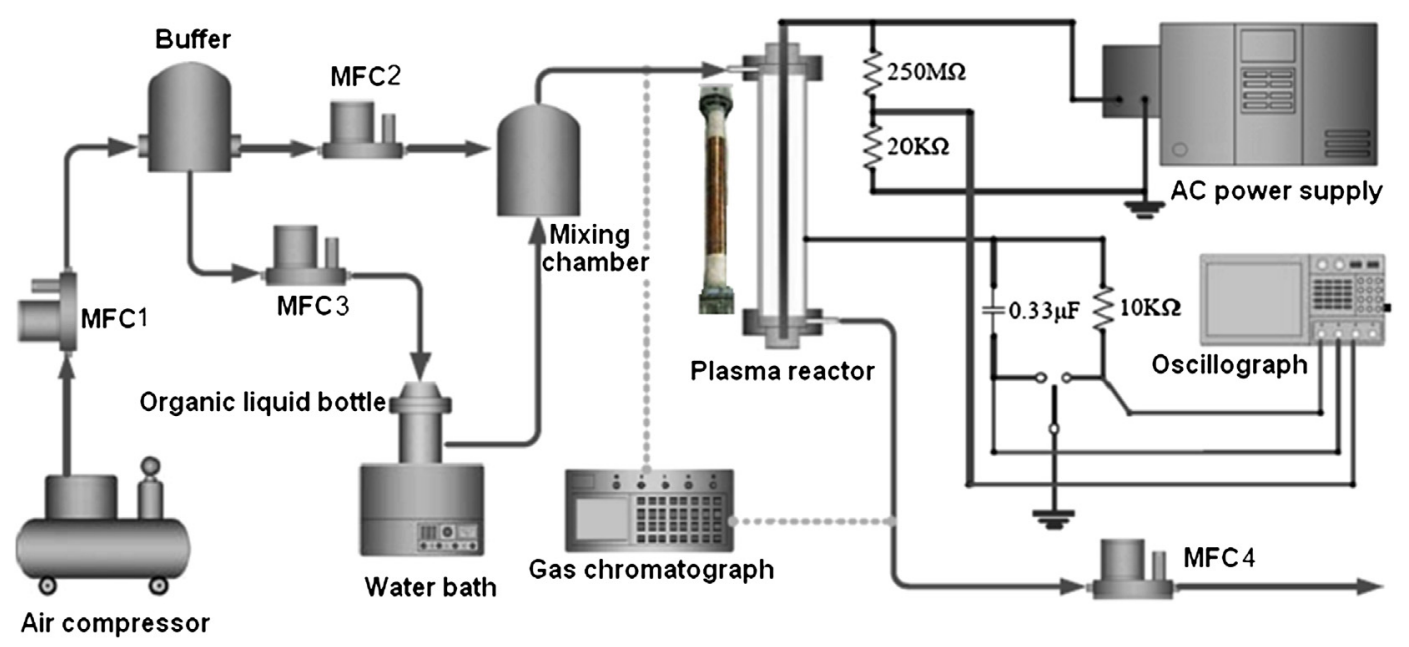

Fig. 1. Schematic diagram of the experimental setup. 

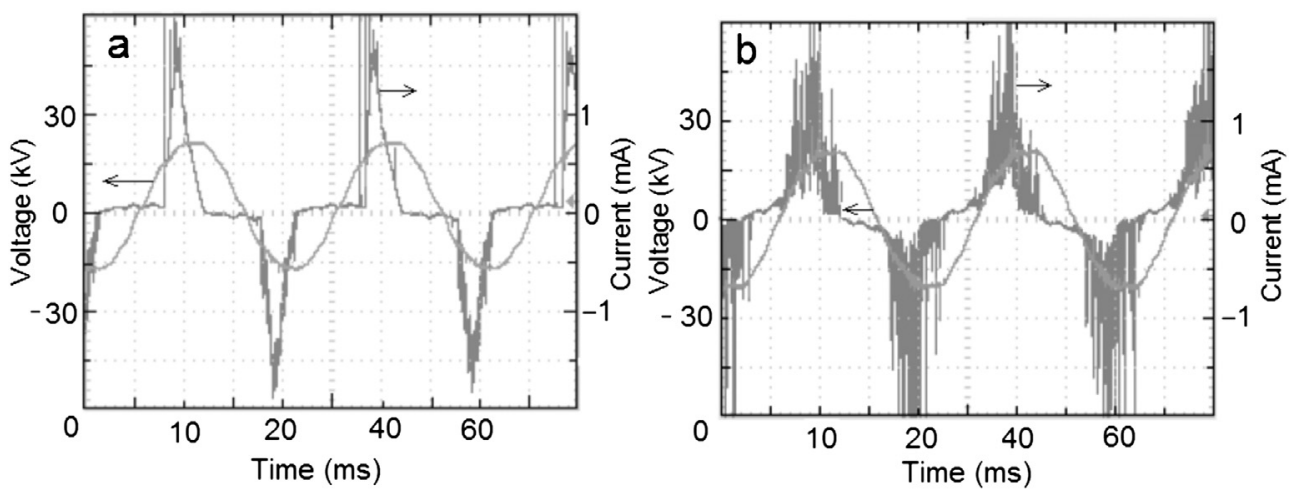

Fig. 2. Voltage and current waveforms in the presence (a) and absence (b) of ceramic Raschig rings at $12 \mathrm{kV}$ applied voltage.

diagram. The Lissajous figure requires the signal of 12,500:1 voltage divider as $x$ input, while requires the voltage signal between the two poles of $C_{\mathrm{m}}$ as $y$ input. In this case, the Lissajous diagram represents a parallelogram, as shown in Fig. 3. When the switch is turned to the right, the time-based current signal can be obtained via the voltage signal between the two poles of $R 3$ divided by $10 \mathrm{k} \Omega$.

In this study, the frequency is set at $50 \mathrm{~Hz}$, which can be regulated from the dial of power supply instrument. The area of the parallelogram conforms to the discharge energy per one cycle, and the average discharge power can be obtained by multiplying the discharge energy per one cycle by AC frequency [16]:

$P=f \cdot C_{\mathrm{m}} \cdot S$

where $C_{\mathrm{m}}=0.33 \mu \mathrm{F}$ is the measuring capacitance, $f=50 \mathrm{~Hz}$ is the AC frequency and $S$ represent the area of parallelogram, respectively.

During the discharge processing, the analysis of equivalent capacitance is another significant consideration except for the energy consumption. The capacitances $\left(C\right.$ and $\left.C_{\mathrm{d}}\right)$ in the equivalent circuit of the reactor can be exhibited by the two gradients of parallelogram for $Q-V$ Lissajous diagram. In particular, the total equivalent capacitance $(C)$ of the dielectric barrier and the gap between electrode and surface of dielectric barrier can be represented by the gradient of line $\mathrm{AB}$ or $\mathrm{CD}(\operatorname{tg} \alpha)$, as shown in Fig. 3 . Here, $C$ can be determined by the following equation:

$C=C_{\mathrm{m}} \cdot \operatorname{tg} \alpha$

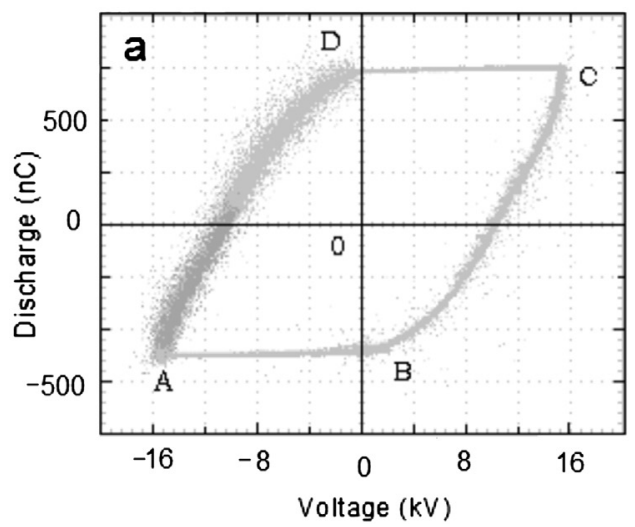

On the other hand, the equivalent capacitance of the dielectric barrier $\left(C_{\mathrm{d}}\right)$ can be represented by the gradient of line $\mathrm{AD}$ or $\mathrm{BC}$ $(\operatorname{tg} \beta)$, and $C_{d}$ can be calculated by the following relation:

$C_{\mathrm{d}}=C_{\mathrm{m}} \cdot \operatorname{tg} \beta$

Consequently, the equivalent capacitance of the gap $\left(C_{\mathrm{g}}\right)$ can be calculated according to the values of barrier capacitance and total capacitance, and the formula for $C_{\mathrm{g}}$ can be expressed as following:

$C_{g}=\frac{C \cdot C_{d}}{C_{d}-C}$

\section{Results and discussion}

\subsection{Discharge characteristics}

Fig. 2 shows the wave forms of applied voltage and current in the presence (Fig. 2a) and absence (Fig. 2b) of the ceramic Raschig rings. Comparison between Fig. 2a and b illustrates the improvement in electrical behavior in the case of padding filling the reaction room of plasma reactor. It can be seen that the current waveform is nonsinusoidal distorted by a number of pulses (microdischarges) superposing on the capacitive wave. These microdischarges, uniformly distributed over the surface of dielectric, are of nanosecond duration and generate radicals, excited atomic, molecular species that initiate plasma chemical reactions. Obviously, the number of microdischarge pulses in the presence of ceramic Raschig rings was much larger than that in the absence of

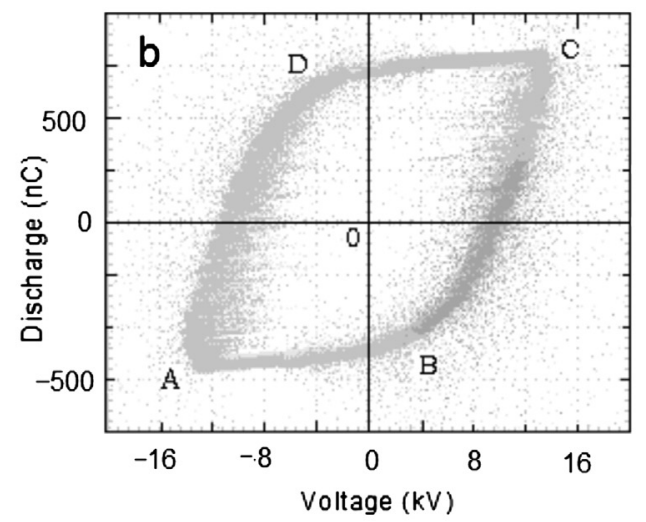

Fig. 3. $V-Q$ Lissajous diagram in the presence (a) and absence (b) of ceramic Raschig ring at $12 \mathrm{kV}$ applied voltage. 


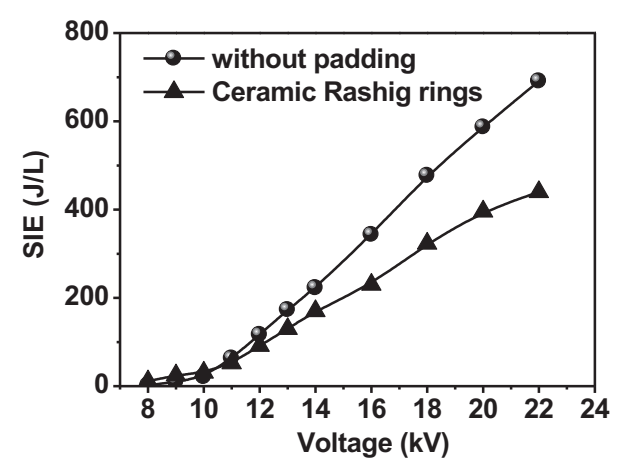

Fig. 4. Variation of SIE as a function of voltage in the presence and absence of ceramic Raschig rings. The standard deviations for SIE are within the range of 1.3-9.7.

padding. Previous studies found that the number of pulses is proportional to the VOC molecular decomposition via NTP [17]. Compared with the NTP reactor without padding, the value of capacitive current appears higher with padding.

The specific input energy (SIE) is defined as the average power dissipated in the discharge divided by the total gas flow rate, and it can be calculated by the discharge power and the gas flow rate. Fig. 4 shows the variation of SIE as a function of applied voltage in the presence and absence of padding respectively. Under low voltage condition $(<11 \mathrm{kV})$, the value of SIE for the reactor with ceramic Raschig rings is slightly higher than that for the empty reactor. With further increasing the voltage $(>11 \mathrm{kV})$, the SIE depends almost linearly on the voltage, however, the value is significantly lower with the existence of padding than that without padding, which means that the padding cuts down the energy consumption. In particular, with increasing the applied voltage from 11 to $22 \mathrm{kV}$, the SIE increases from 61 to $692 \mathrm{~J} / \mathrm{L}$ for the empty reactor, and increases from 52 to $440 \mathrm{~J} / \mathrm{L}$ for the reactor with ceramic Raschig rings padding. When the voltage is low, the dielectric paddings have not develop their functions effectively yet, and the type of plasma mainly refers as corona discharge which occurs in a region of high electric field near electrically stressed wire edges. Therefore, the space charge can transfer more easily along the surface of padding, which leads to greater current and discharge power. When the voltage is high enough, it is developing into dielectric barrier discharge which polarizes the dielectric padding and forms intense electric field around each edge and point with small curvature radius, resulting in micro-discharges and initiating chemical reactions throughout the entire discharge volume.

In the experiment, the plasma reactor can be equivalent to a capacitive load. The methods of calculating the equivalent capacitance of gap and the equivalent capacitance of dielectric barrier by $Q-V$ Lissajous diagram were given above. The equivalent gap capacitance as a function of applied voltage for different dielectric conditions inside the reactor are shown in Fig. 5. It was found that $C_{\mathrm{g}}$ decreases gradually with increasing the applied voltage for the two cases, similar to that previously reported $C_{\mathrm{g}}$ variation [18]. Take no-padding reactor for example, when the applied voltage increases from 10 to $16 \mathrm{kV}, C_{\mathrm{g}}$ decreases from 16 to $13 \mathrm{pF}$. Obviously, the values of $C_{g}$ are higher for ceramic Raschig ring reactor compared with that for the empty tube, $21 \mathrm{pF}$ and $14 \mathrm{pF}$ respectively at $12 \mathrm{kV}$. It is known that the gap capacitance is directly proportional to the dielectric constant $\left(\varepsilon_{\mathrm{g}}\right)$ and inversely proportional to the gap length $\left(L_{\mathrm{g}}\right), C_{\mathrm{g}} \propto \varepsilon_{\mathrm{g}} / L_{\mathrm{g}}$. As a consequent, for a given $\varepsilon_{\mathrm{g}}$, greater $C_{\mathrm{g}}$ can be obtained by smaller gap length. With respected to the NTP reactor filling with ceramic Raschig rings, the padding takes up much space between the inner electrode and the surface of dielectric, which results in smaller $L_{\mathrm{g}}$ and bigger $C_{\mathrm{g}}$.

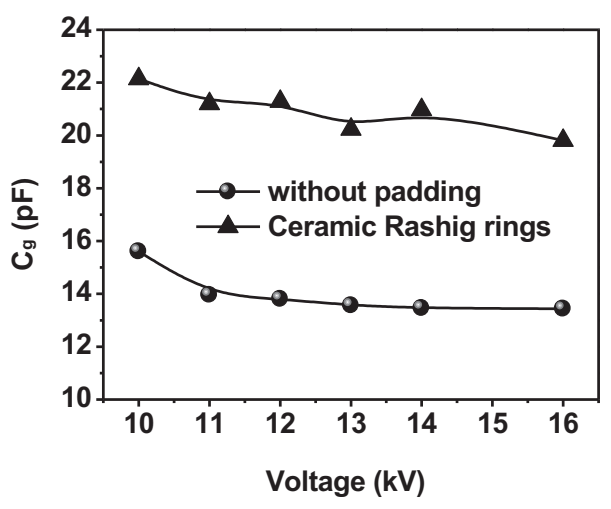

Fig. 5. Equivalent gap capacitance as a function of voltage in the presence and absence of ceramic Raschig rings. The standard deviations for equivalent gap capacitance are within the range of $0.1-0.8$.

The variety of equivalent capacitance of the dielectric barrier obtained by using $Q-V$ Lissajous diagrams as a function of voltage is shown in Fig. 6. For the empty plasma reactor, $C_{\mathrm{d}}$ initially increases remarkably from 338 to $652 \mathrm{pF}$ with increasing applied voltage in the range of $10-12 \mathrm{kV}$, and then stabilizes at $700 \mathrm{pF}$ approximately with further increasing of voltage. Despite of certain micro-error, $C_{\mathrm{d}}$ follows a similar trend for the ceramic Raschig rings reactor. The trend of $C_{\mathrm{d}}$ for the applied voltage can be explained by the characteristics of ceramic tube's permittivity, which exhibit the similar variation tendency to the value of $C_{\mathrm{d}}$ versus electric field.

\subsection{Toluene removal}

According to the related theories of plasma, free electrons transfer all or part of their kinetic energy to the molecules through inelastic collisions with the target molecules which are then dissociated and oxidated into $\mathrm{CO}_{2}$ and $\mathrm{H}_{2} \mathrm{O}$. Fig. 7 demonstrates the influence of ceramic Raschig rings packing on toluene decomposition compared with the empty reactor as a function of SIE. It is observed that the toluene removal process assisted by some ceramic Raschig rings is significantly enhanced. When the SIE varies in the range of 53-445 J/L, toluene removal ratio increase from $26 \%$ to $97 \%$ with Raschig rings packing, while increase from $23 \%$ to $48 \%$ without packing. The results suggest that the padding plays an important role in the process of toluene decomposition. The improvement in toluene conversion can be attributed to the enhancement of intensity of electric field in the presence of ceramic Raschig rings, which is in accordance with the other researchers

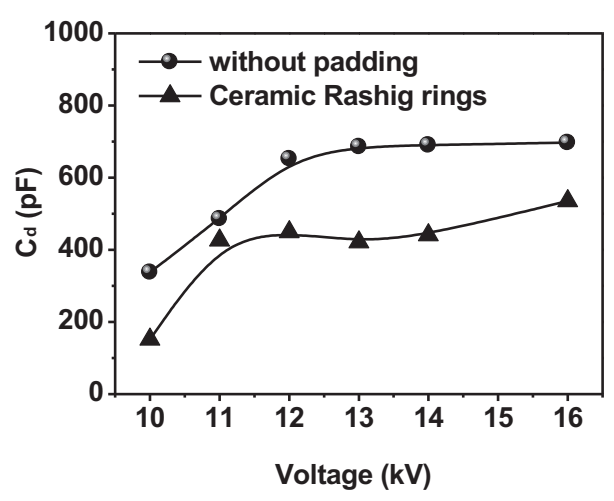

Fig. 6. Equivalent dielectric barrier capacitance as a function of voltage in the presence and absence of ceramic Raschig rings. The standard deviations for Equivalent dielectric barrier capacitance are within the range of 4.6-21.2. 


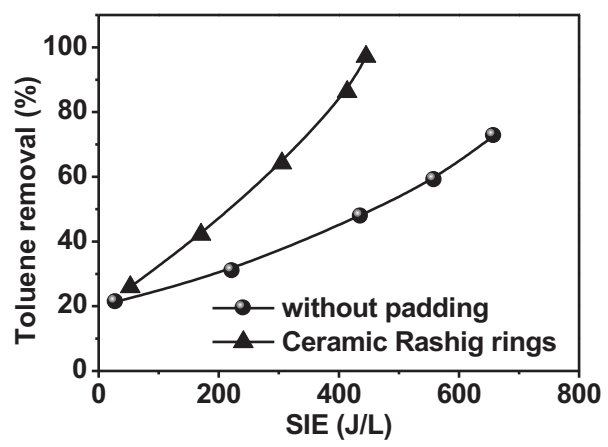

Fig. 7. Toluene removal as a function of SIE in the presence and absence of ceramic Raschig rings. The standard deviations for toluene removal are within the range of 0.2-1.3.

[19]. Based on the above discussion, it is evidenced that the removal efficiency of toluene treated by NTP process can be significantly improved by the introduction of some dielectrics inside the reactor.

\subsection{Energy yield for toluene removal}

The energy yield is usually used to evaluate the system performance of NTP process [20]. The energy yield for toluene removal was also analyzed in this study and it can be described by the following equation:

$\mathrm{EY}(\mathrm{g} / \mathrm{kWh})=\frac{\left(C_{\text {in }}-C_{\text {out }}\right) \times Q_{\mathrm{g}}}{P}$

where $Q_{g}$ and $P$ are the gas flow rate and the discharge power $(W)$, respectively, and $C_{\text {in }}$ and $C_{\text {out }}$ indicate inlet and outlet average concentrations of toluene, respectively.

Fig. 8 presents the energy yield for toluene removal as a function of removal ratio in the presence and absence of ceramic Raschig rings. As seen in the Figure, the energy yield decreases initially with the increase in toluene removal ratio. Then, with the ratio exceeding 50\%, the toluene removal energy yield stabilizes at about $10 \mathrm{~g} / \mathrm{kWh}$ in the presence of padding, which is 2 times higher than that of $5 \mathrm{~g} / \mathrm{kWh}$ in the absence padding. The energy yield of the padding filling system for the decomposition of toluene exhibits high level compared with the previous studies [21,22], which can be attributed to the high reactive plasmas generated by corundum ceramic reactor combined with ceramic Raschig rings possessing high permittivity. Furthermore, it can be concluded that the introduction of ceramic Raschig rings inside the reactor improves the energy yield significantly, and it lowers the energy consumption consequently.

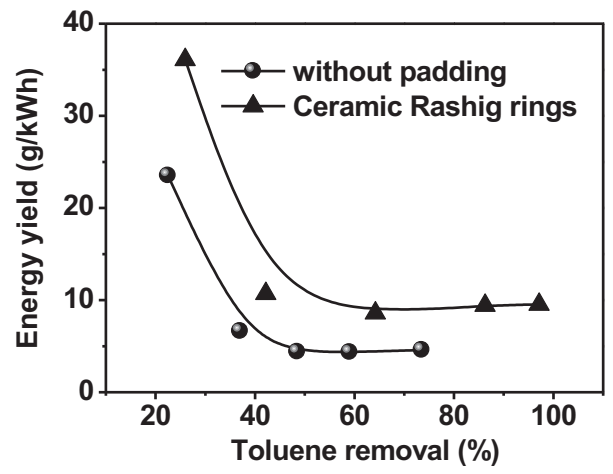

Fig. 8. Energy yield for toluene removal as a function of removal ratio in the presence and absence of ceramic Raschig rings. The standard deviations for energy yield are within the range of $0.3-1.0$

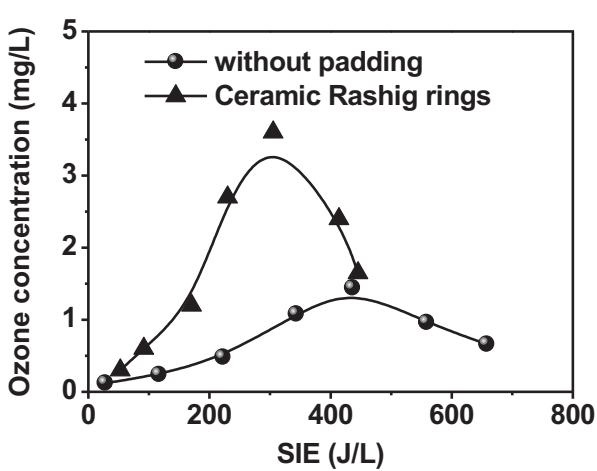

Fig. 9. Ozone concentration as a function of SIE in the presence and absence of ceramic Raschig rings. The standard deviations for ozone concentration are within the range of $0.01-0.14$.

\subsection{Ozone formation in the non-thermal plasma reactor}

As already observed, non-thermal plasma produces ozone which is customarily considered as an important inorganic byproduct by the ionization of oxygen molecules from air. The ozone is generated through the reaction between an oxygen molecule and atomic oxygen which is generated by electron collisions on $\mathrm{O}_{2}$. The reaction is expressed as following:

$\mathrm{e}+\mathrm{O}_{2} \rightarrow 2 \mathrm{O}+\mathrm{e}$

$\mathrm{O}+\mathrm{O}_{2}+\mathrm{M} \rightarrow \mathrm{O}_{3}+\mathrm{M}$

where $\mathrm{M}$ can be $\mathrm{N}_{2}$ or $\mathrm{O}_{2}$ in air [23]. The variations of the ozone concentration in the effluent gas and the toluene abatement as a function of SIE are illustrated in Fig. 9. By increasing with the input energy in the range of 30-660 J/L, for no-padding plasma reactor, the concentration of ozone initially increases from $0.12 \mathrm{mg} / \mathrm{L}$ to $1.44 \mathrm{mg} / \mathrm{L}$, and then passes through a maximum of $1.44 \mathrm{mg} / \mathrm{L}$ before a rapid decrease with further increase in SIE. Similarly, the variation of ozone concentration follows the same tendency for the existence of ceramic Raschig rings. Actually, the tendency is in consistent with previous reports on the ozone generation during the plasma process to decompose VOCs [24,25]. At the same time, as mentioned above, toluene removal ratio increases monotonically with the increase of SIE, and it can be concluded that ozone formed in plasma reactor is not involved in the toluene decomposition, similar to previous investigations [26,27]. The results of ozone generation indicate that the applied power must high enough to yield adequate collection efficiency but not so high as to produce hazardous levels of ozone.

\section{Conclusions}

The abatement of toluene with dielectric barrier discharges was experimentally investigated through a comparison between in presence and absence of ceramic Raschig rings padding inside the coaxial cylindrical reactor. Furthermore, electrical parameters during plasma processing to decompose toluene were analyzed by using the $Q-V$ Lissajous diagrams. The obtained results showed that, at high-voltage operation $(>11 \mathrm{kV})$, the SIE depended almost linearly on the voltage and the value was significantly lower in case of ceramic Raschig rings padding than that in the absence of padding. $C_{\mathrm{g}}$ decreased with increasing voltage and the existence of padding, while $C_{\mathrm{d}}$ increased initially and stabilized at about $700 \mathrm{pF}$ with further increase in voltage. In contrast to no-padding reactor, toluene conversion was enhanced by dielectric characteristic of 
ceramic Raschig rings padding, especially under high input energy condition, $97 \%$ against $48 \%$ at SIE $445 \mathrm{~J} / \mathrm{L}$. With respect to the energy yield for toluene removal, in presence of padding, the efficiency was significantly improved up to $10 \mathrm{~g} / \mathrm{kWh}$, which was 2 times higher than the efficiency $5 \mathrm{~g} / \mathrm{kWh}$ in the absence of padding with removal ratio exceeding $50 \%$.

\section{Acknowledgment}

This study was financially supported by National Natural Science Foundation of China (21307088, 51206119).

\section{References}

[1] T. Hakoda, A. Shimada, A. Kimura, M. Taguchi, Y. Sugo, K. Araki, E.B. Dally, K. Hirota, An electron-beam irradiation/catalytic oxidation system for purification of aromatic hydrocarbons/air mixture under practical gas-flow condition, Ind. Eng. Chem. Res. 49 (2010) 5517-5522.

[2] B. Dou, J. Li, Q. Hu, C. Ma, C. He, P. Li, Z. Hao, S. Qiao, Hydrophobic micro/mesoporous silica spheres assembled from zeolite precursors in acidic media for aromatics adsorption, Microporous Mesoporous Mater. 133 (2010) 115-123.

[3] M. Bannai, A. Houkabe, M. Furukawa, T. Kashiwagi, A. Akisawac, T. Yoshidad, H. Yamadae, Development of efficiency-enhanced cogeneration system utilizing high-temperature exhaust-gas from a regenerative thermal oxidizer for waste volatile-organic-compound gases, Appl. Energy 83 (2006) 929-942.

[4] J.F. Nastaj, B. Ambrożek, J. Rudnicka, Simulation studies of a vacuum and temperature swing adsorption process for the removal of VOC from waste air streams, Int. Commun. Heat Mass Transfer 33 (2006) 80-86.

[5] S. Salvador, J.M. Commandré, Y. Kara, Thermal recuperative incineration of VOCs: CFD modelling and experimental validation, Appl. Therm. Eng. 26 (2006) 2355-2366.

[6] P. Dwivedi, V. Gaur, A. Sharma, N. Verma, Comparative study of removal of volatile organic compounds by cryogenic condensation and adsorption by activated carbon fiber, Sep. Purif. Technol. 39 (2004) 23-37.

[7] B. Michel, H. Stéphane, A critical look at the air infiltration term in the Canadian energy rating procedure for windows, Energy Buildings 37 (2005) 997-1006.

[8] L. Xia, L. Huang, X. Shu, R. Zhang, W. Dong, H. Hou, Removal of ammonia from gas streams with dielectric barrier discharge plasmas, J. Hazard. Mater. 152 (2008) 113-119.

[9] M. Magureanu, N.B. Mandache, V.I. Parvulescu, Ch Subrahmanyam, A. Renken, L. Kiwi-Minsker, Improved performance of non-thermal plasma reactor during decomposition of trichloroethylene: optimization of the reactor geometry and introduction of catalytic electrode, Appl. Catal. B Environ. 74 (2007) 270-277.

[10] C. Ayrault, J. Barrault, N. Blin-Simiand, F. Jorand, S. Pasquiers, A. Rousseau, J.M. Tatibouët, Oxidation of 2-heptanone in air by a DBD-type plasma generated within a honeycomb monolith supported Pt-based catalyst, Catal Today 89 (2004) 75-81.

[11] A.M. Vandenbroucke, R. Morent, N. De Geyter, C. Leys, Non-thermal plasmas for non-catalytic and catalytic VOC abatement, J. Hazard. Mater. 195 (2011) 30-54.

[12] R. Li, Q. Tang, S. Yin, T. Sato, Plasma catalysis for $\mathrm{CO}_{2}$ decomposition by using different dielectric materials, Fuel Process. Technol. 87 (2006) 617-622.

[13] M.A. Malik, X. Jing, Catalyst assisted destruction of trichloro ethylene and toluene in corona discharges, J. Environ. Sci. 12( (2000) 7-11.

[14] K. Takaki, K. Urashima, J.S. Chang, Ferro-electric pellet shape effect on $\mathrm{C}_{2} \mathrm{~F}_{6}$ removal by a packed-bed-type nonthermal plasma reactor, IEEE Trans. Plasma Sci. 32 (2004) 2175-2183.

[15] C. Subrahmanyam, A. Renken, L. Kiwi-Minsker, Novel catalytic nonthermal plasma reactor for the abatement of VOCs, Chem. Eng. J. 134 (2007) 78-83.

[16] T.C. Manley, The electric characteristics of the ozonator discharge, J. Electrochem. Soc. 84 (1943) 83-96.

[17] M. Moscosa-Santillan, A. Vincent, E. Santirso, J. Amouroux, Design of a DBD wire-cylinder reactor for NOx emission control: experimental and modelling approach, J. Cleaner Prod. 16 (2008) 198-207.

[18] K. Takaki, M. Shimizu, S. Mukaigawa, Effect of electrode shape in dielectric barrier discharge plasma reactor for NOx removal, IEEE Trans. Plasma Sci. 32 (2004) 32-38.

[19] B.Y. Lee, S.H. Park, S.C. Lee, M. Kang, S.J. Choung, Decomposition of benzene by using a discharge plasma-photocatalyst hybrid system, Catal. Today 93 (2004) 769-776.

[20] H.H. Kim, G. Prieto, K. Takashima, S. Katsura, A. Mizuno, Performance evaluation of discharge plasma process for gaseous pollutant removal, J. Electrost. 55 (2002) 25-41.

21] T. Ohkubo, D. Li, D. Yakushiji, S. Kanazawa, Y. Nomoto, Decomposition of VOC in air using a streamer corona discharge reactor combined with catalysts, J. Adv. Oxid. Technol. 6 (2003) 75-79.

[22] K. Inoue, H. Okano, Y. Yamagata, K. Muraoka, Y. Teraoka, Performance tests of newly developed adsorption/plasma combined system for decomposition of volatile organic compounds under continuous flow condition, J. Environ. Sci. 23 (2011) 139-144.

23] M. Magureanu, N.B. Mandache, P. Eloy, E.M. Gaigneaux, V.I. Parvulescu, Plasma-assisted catalysis for volatile organic compounds abatement, Appl. Catal. B Environ. 61 (2005) 12-20.

[24] T. Oda, Non-thermal plasma processing for environmental protection: decomposition of dilute VOCs in air, J. Electrost. 57 (2003) 293-311.

[25] T. Yamamoto, VOC Decomposition by nonthermal plasma processing-a new approach, J. Electrost. 42 (1997) 227-238

26] S. Futamura, H. Einaga, H. Kabashima, L. Hwan, Synergistic effect of silen discharge plasma and catalysts on benzene decomposition, Catal. Today 89 (2004) 89-95.

[27] C. Subrahmanyam, A. Renken, L. Kiwi-Minsker, Catalytic abatement of volatile organic compounds assisted by non-thermal plasma: part II. Optimized catalytic electrode and operating conditions, Appl. Catal. B Environ. 65 (2006) 157-162. 